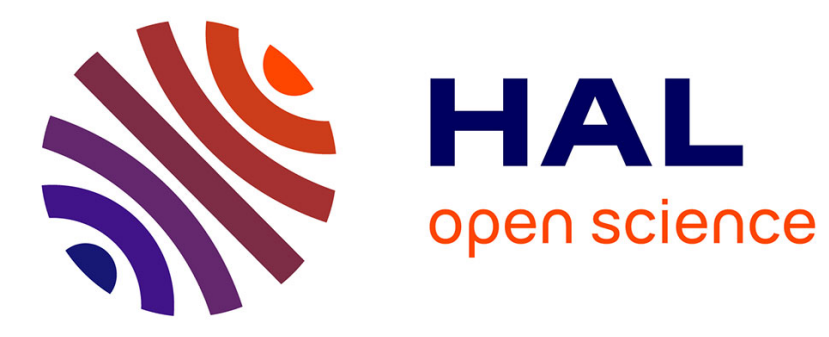

\title{
Empirische Sozialforschung und ihre Macher
}

Sacha Raoult

\section{To cite this version:}

Sacha Raoult. Empirische Sozialforschung und ihre Macher: Die soziale Produktion des Wissens über die Wirksamkeit der Todesstrafe . Soziale Probleme, 2015, 10.1007/s41059-015-0004-x . hal01310212

\section{HAL Id: hal-01310212 \\ https://hal-amu.archives-ouvertes.fr/hal-01310212}

Submitted on 2 May 2016

HAL is a multi-disciplinary open access archive for the deposit and dissemination of scientific research documents, whether they are published or not. The documents may come from teaching and research institutions in France or abroad, or from public or private research centers.
L'archive ouverte pluridisciplinaire $\mathbf{H A L}$, est destinée au dépôt et à la diffusion de documents scientifiques de niveau recherche, publiés ou non, émanant des établissements d'enseignement et de recherche français ou étrangers, des laboratoires publics ou privés.

$$
\text { Copyright }
$$




\title{
Empirische Sozialforschung und ihre Macher Die soziale Produktion des Wissens über die Wirksamkeit der Todesstrafe
}

\author{
von Sacha Raoult ${ }^{\text {* }}$
}

\section{Zusammenfassung}

Die Forschung zur abschreckenden Wirkung der Todesstrafe hat eine in ihrem Umfang beeindruckende Masse an Veröffentlichungen hervorgebracht, deren Aussagen mitunter verwirrend und widersprüchlich sind. Eine Untersuchung der Forschergruppen, die sich mit der Korrelation zwischen Vollstreckungen der Todesstrafe und Tötungsdelikten befassen ergibt, dass es eine "lautstarke Minderheit“ von Untersuchungen gibt, die sich stark für die Todesstrafe aussprechen. Dabei zeigt es sich, dass die Person des Autors eines Aufsatzes zuverlässigere Vorhersagen über die dort zu findenden Korrelationen erlaubt als die verwendeten Daten. Es lässt sich also ein Profil für Autoren erstellen, die häufig zu Ergebnissen kommen, die zugunsten der Todesstrafe sprechen: Es handelt sich um amerikanische Wirtschaftswissenschaftler, die sich selbst nicht als Spezialisten für Verbrechens- und Strafforschung beschreiben und die an einer weniger angesehenen Fakultät als der unterrichten, an der sie promoviert haben. Im Gegensatz dazu kommen Soziologen und Ökonomen an angesehenen Universitäten eher zu dem Ergebnis, dass die Todesstrafe keine Wirkung auf die Mordrate habe.

\section{Einleitung}

Einer der wichtigsten Beiträge zum Denken des 20. Jahrhunderts ist sicherlich die Einsicht in die „Bedeutung des Blicks“ für die „,betrachtete Sache“ (Gide 2012). Die bedeutendsten Wissenschaftshistoriker haben die Vorstellung entwickelt, dass wissenschaftliche Arbeit bestimmt wird vom spezifi-

* Zuerst erschienen 2015 als Des méthodes et des hommes. La production sociale du savoir sur l'effet dissuasif des peines. Déviance \& Société mars, 39/1: 99-120. Übersetzung: Michael G. Esch, Bearbeitung: Axel Groenemeyer. 
schen Paradigma, in dessen Rahmen sie betrieben wird (Kuhn 1967), und damit von den sozialen Bedingungen ihrer epistemischen Möglichkeit (Foucault 2014).

In der Soziologie richtet sich das Hauptaugenmerk in diesem Bereich von unterschiedlichen Ausgangspunkten aus auf die Frage nach dem Prozess der „Herstellung“ wissenschaftlicher Tatsachen, wobei der Subjektivität der Forschenden und dem Prozess der Konstruktion wissenschaftlichen Wissen jeweils mehr oder weniger Platz eingeräumt wurde (siehe z. B. Abbott 2001; Barnes/Bloor/Henry 1996; Latour/Woolgar 2006). Gleichzeitig haben sich Spezialisten und Spezialistinnen der verschiedensten Disziplinen die Frage gestellt, in welchem Maße eine Verzerrung wissenschaftlicher Ergebnisse durch die Person der Forscher und Forscherinnen feststellbar ist (Bresnick 2008; Vaccaro/Patel/Fisher 2011), und wurden statistische Werkzeuge vorgestellt, mit denen sich derartige Autorenbias in den Veröffentlichungen feststellen lassen (Egger et al. 1997). Diese Debatte hatte durchaus Auswirkungen auf die Organisation der Forschung und hat z.B. dazu beigetragen, gröBere Transparenz in den Beziehungen zwischen Forschern und Wirtschaft, insbesondere in den Naturwissenschaften, herzustellen (Bekelman/Li/Gross 2003; Thompson 1993).

Selten sind dagegen Versuche, Autorenbias in den Sozial- und Humanwissenschaften - und namentlich in den Kriminalwissenschaften - über die Frage finanzieller Interessenkonflikte hinaus systematisch zu messen. Es lässt sich die Hypothese formulieren, dass besonders bei so stark politisierten Fragen wie etwa der Wirksamkeit der Todesstrafe, durch Abschreckung Morde $\mathrm{zu}$ verhindern, Haltungen und Einstellungen des Autors oder der Autorin, sein und ihr Werdegang, die Ausbildung und Interessengebiete eine Voraussage über einen Teil seiner oder ihrer Ergebnisse erlauben.

Um die Gültigkeit dieser Hypothese zu überprüfen. stützt sich der vorliegende Aufsatz auf eine jüngere Erhebung über alle Studien zur Todesstrafe seit den 1970er Jahren (Yang/Lester 2008). Diese Erhebung zählt 100 Arbeiten, von denen 85 Prozent die Frage beantworten wollen: „Welche Korrelation besteht zwischen Hinrichtungen und Mord/Totschlag?“" Wir wollen in dieser Erhebung drei Dinge untersuchen. Zunächst soll gefragt werden, ob die Ergebnisse der Arbeiten zur Todesstrafe sich gemäß der Gaußschen Normalverteilung gruppieren, das heißt, ob es ebenso viele veröffentlichte Ergebnisse oberhalb wie unterhalb der Mittellinie gibt und ob die „Extremfälle“ 
seltener sind als die „durchschnittlichen“ Ergebnisse. Diese Methode wurde gewählt, weil es sich um einen klassischen Test zur Feststellung von Veröffentlichungstrends handelt; seine Aussagekraft wird im Weiteren noch erklärt werden. Der zweite Schritt der Arbeit besteht in einer Messung des Zusammenhangs zwischen den Ergebnissen einer Publikation und den Ergebnissen der folgenden Veröffentlichung der gleichen Gruppe von Forschern und Forscherinnen. Dabei wird der Prädiktionswert der vorangegangen Veröffentlichung mit dem Prädiktionswert der Art der verwendeten Daten verglichen (also mit einer häufigen Erklärung der Unterschiede in den Einschätzungen der Todesstrafe). Schließlich erstellt der vorliegende Aufsatz wissenschaftsbiographische Profile von Forscherinnen und Forschern auf der Grundlage der jeweiligen Ergebnisse. Dazu wurde eine systematische Analyse aller Elemente vorgenommen, die sich den Lebensläufen der Autoren und Autorinnen entnehmen ließen. Einige Elemente wie der Fachbereich, das Land, die Einstufung der Universität, an der zuletzt gearbeitet wurde, sowie die Interessengebiete stehen offensichtlich in engem Zusammenhang damit, ob jemand Arbeiten für oder gegen die Wirksamkeit der Todesstrafe hervorbringt. Schließlich werden einige Wege vorgeschlagen, auf denen sich der Aussagewert unserer Ergebnisse vertiefen ließe.

\section{2. Über die Natur eines akademischen Streits}

Es lassen sich mindestens 800 internationale Veröffentlichungen finden, die sich bemühen, einen möglichen Abschreckungseffekt von Strafen zu messen (Dölling et al. 2009; Yang/Lester 2008). Einige dieser Studien argumentieren, Kriminelle handeln insofern rational, als sie mit der Härte, der Wahrscheinlichkeit und/oder der Schnelligkeit der Strafe umgehen, als sei sie ein „Preis“, den sie angesichts des möglichen Gewinns, den sie aus der Gesetzesübertretung zu ziehen hoffen, zu zahlen bereit seien oder nicht. Die empirischen Arbeiten zur Wirksamkeit der Todesstrafe scheinen dann das Modell des rationalen Delinquenten, das der Wirtschaftswissenschaftler Gary Becker 1968 vorgeschlagen hat, zu bestätigen. Andere Arbeiten kommen eher zu der entgegengesetzten Schlussfolgerung, derzufolge die Aussicht auf eine empfindliche Strafe keinerlei Auswirkungen auf das Verhalten einer Person habe und das Abschreckungsmoment der Strafe, ,sofern es überhaupt besteht, eine 
Bedeutung einnimmt, die weit von den Erwartungen des Modells entfernt ist" (Paternoster 2010).

Die Todesstrafe selbst war zwischen 1970 und 2013 Gegenstand von mehr als 100 Arbeiten. Einige Studien erkennen einen deutlichen Abschreckungseffekt der Todesstrafe „selbst bei Verbrechen aus Leidenschaft“ (Rubin 2006), und ihre Autoren gehen so weit zu sagen, dass bis zu 18 Leben je Hinrichtung gerettet würden. Weitaus zahlreicher sind allerdings Studien, die keine statistisch signifikante Korrelation zwischen Hinrichtungen und Mord/ Totschlag finden; die Autoren und Autorinnen neigen daher eher zu dem Schluss , dass die Todesstrafe einen „Nulleffekt“ auf das Verbrechen ausübe. Eine dritte Gruppe von Studien findet eine so genannte „brutalisierende“ Wirkung der Todesstrafe, das heißt, sie stellen eine positive Korrelation zwischen der Zahl der Hinrichtungen und der Zahl der Gewaltverbrechen fest. Ein solches Verhältnis entspricht dem berühmten Argument Beccarias, demzufolge die Todesstrafe zur Folge habe, den Tod zu banalisieren und „einen Menschen mit verbrecherischen Neigungen“ zu Trugschlüssen zu verleiten (1998/1851: 50).

Zwar mag diese Disparität der Ergebnisse einen Laien erstaunen, an und für sich ist sie jedoch nicht überraschend. Unterschiedliche Studien, die in unterschiedlichen Kontexten mit verschiedenen Methoden und Datenbeständen betrieben werden, können schon aufgrund von Zufällen zu unterschiedlichen Resultaten kommen. Was die Todesstrafe angeht sind verschiedene Erklärungsmodelle für diese Disparitäten vorgeschlagen worden. Keine dieser Erklärungen wird allgemein akzeptiert; wir werden hierauf noch zurückkommen. Unser erster Eindruck ist allerdings, dass es kein großes Geheimnis aufzuklären gäbe, wenn die Disparität der Ergebnisse die einzige Form von Heterogenität wäre, die sich in diesen quantitativen Untersuchungen findet. Es werden natürlich nicht die gleichen Gegenstände betrachtet, und es werden nicht die gleichen Aspekte berücksichtigt. Weitaus schwerer ist dagegen $\mathrm{zu}$ verstehen, wieso sich diese Zersplitterung der Schlussfolgerungen auf einer anderen Ebene der akademischen Welt wiederfinden lässt.

(1) Disparitäten im Forschungsstand. Quantitative Studien. die in referierten Zeitschriften erscheinen, werden häufig mit einem „Forschungsstand“ eingeleitet, der die bis dahin erschienene einschlägige Literatur zusammenfasst. Während aber unterschiedliche Studien aufgrund ihrer methodologischen Anlage oder dem Forschungskontext durchaus unterschiedliche Ergeb- 
nisse erbringen können, gibt es keinen einsehbaren Grund, warum sich die Forschungsstände bei mehreren Artikeln aus der gleichen Zeit unterscheiden sollten. Theoretisch haben alle Forscher und Forscherinnen Zugang zur gleichen Literatur. Wenn wir die Forschung betrachten, die sich auf die Feststellung der Korrelation zwischen der Zahl der Hinrichtungen und der Zahl der Gewaltverbrechen ${ }^{3}$ beschränkt, so fallen sofort zwei Extreme in den „Forschungsständen“ auf. Zhiqiang Liu fasst die Literatur in seinem Aufsatz $\mathrm{Ca}$ pital Punishment and the Deterrence Hypothesis: Some New Insights and Empirical Evidence im Eastern Economic Journal aus dem Jahre 2004 wie folgt zusammen: „Im Wesentlichen haben die Ökonomen eine statistisch signifikante negative Korrelation - das heißt eine abschreckende Wirkung zwischen Hinrichtungen und Morden festgestellt" (S. 237). Ganz im Gegensatz dazu leiten Zimring, Fagan und Johnson ihre eigene, wenige Jahre später publizierte Arbeit so ein: „Die verfügbaren Daten (über die Todesstrafe) sind schwach, großenteils weil es da, wo sie in erster Linie untersucht wird, so wenige Hinrichtungen gibt“ (2010: 1). „Es lässt sich kein irgend gearteter Abschreckungseffekt in den Vereinigten Staaten feststellen" (S. 2).

Es ist keine harmlose Feststellung, dass die Disparitäten in den „Forschungsständen" zu einem großen Teil die Unterschiedlichkeit der Resultate widerspiegeln. Der typische Aufsatz präsentiert seine Schlussfolgerungen als Fortsetzung der vorliegenden Literatur und nicht im Gegensatz dazu: So viel hat eine Forschungsgruppe für die Gesamtheit der Studien zum Abschreckungseffekt der Todesstrafe feststellen können. Dölling et al. (2009: 206) zufolge präsentieren 70 Prozent der Artikel in ihrer Einleitung einen Forschungsstand, der mit den Schlussfolgerungen des Aufsatzes übereinstimmt. Wir befinden uns hier im Bereich dessen, was Thomas S. Kuhn „Normalwissenschaft" genannt hat, also einer Forschung, die im Wesentlichen darauf beruht, ein herrschendes Paradigma zu bestätigen (Kuhn 1967), wobei es dann allerdings mehrere konkurrierende Normalitäten gäbe. Wir werden auf diese Hypothese noch zurückkommen.

(2) Disparitäten in den Aussagen der Experten. Angesichts einer solchen Kakophonie in einer schwer verdaulichen und häufig hoch mathematisierten quantitativen Literatur ist davon auszugehen, dass der Gesetzgeber sich an „Autoritäten“ wenden muss, um die Dinge klarer zu sehen, wenn es sich um eine offensichtlich so grundlegende Frage wie die nach der Wirksamkeit der Todesstrafe für die Verhinderung von Morden handelt. Es ist wenig überra- 
schend, dass solche Expertisen an anderem Ort die gleichen Schemata reproduzieren wie die Forschungsliteratur, häufig mit den gleichen Akteuren, da es im Wesentlichen die Autoren empirischer Untersuchungen sind, die als Experten und Expertinnen herangezogen werden, und zwar nicht nur zu ihren eigenen Arbeiten, sondern über das gesamte Forschungsfeld, dem sie angehören.

Betrachten wir zwei Beispiele solcher entgegengesetzten Expertenmeinungen. Im Laufe des Jahres 2011 beriet das Abgeordnetenhaus von Connecticut über das Gesetz Nr. 1035 zur „Abschaffung der Todesstrafe und ihre Ersetzung durch lebenslange Haft ohne Möglichkeit der bedingten Entlassung" für besonders verwerfliche Morde. Der erste Experte, John Donohue, empfiehlt ausgehend von einem Bericht über die Literatur, einen Teil des für die Todesstrafe vorgesehenen Budgets in die Rekrutierung von Polizisten umzuleiten: „Anders als bei der Todesstrafe konnte gezeigt werden, dass Investitionen in die Polizei eine Möglichkeit sind, die sozialen Kosten von Verbrechen - einschließlich Tötungsdelikten - zu senken" (Connecticut Judiciary Committee 2011). Vor der gleichen Kommission äußerte sich Keri O`Creene mit genau entgegengesetzten Begriffen und ebenso direkt zusammengefasst im Bericht zur Unterstützung der Vorlage: „Dreißig Jahre Forschung haben gezeigt, dass die Todesstrafe Leben rettet.“ Denjenigen, die sich mit den Codes in dieser Berufswelt auskennen, wird sofort auffallen, dass die beiden Vortragenden nicht den gleichen sozialen Status in der akademischen Gemeinschaft genießen: Der erste Sachverständige der Kommission ist ein berühmter Stanford-Professor, während es uns unmöglich gewesen ist, Informationen über die zweite Person zu erhalten. Diese Differenz in der Geltung innerhalb der Universität ist nicht bloß anekdotisch, und wir werden weiter unten sehen, dass sie tatsächlich ein allgemeineres Phänomen illustriert.

Zweites Beispiel: Das Senate Judiciary Committee on the Constitution, Civil Rights, and Property Rights der Vereinigten Staaten von Amerika im Jahre 2006. Vor dieser Kommission trägt Paul Rubin, Professor of Economics and Law an der Emory-Universität ein Gutachten unter dem Titel „Statistischer Beweis über die Todesstrafe und die Abschreckung von Tötungsdelikten“" vor. Dieses Gutachten umfasst 3000 Wörter und wird ergänzt durch eine Bibliographie von dreißig Titeln. Man staunt angesichts der Leichtigkeit, mit der Paul Rubin Ordnung in die Unterschiedlichkeit der zur Todesstrafe veröffentlichten Titel bringt: „Fast alle modernen Studien und alle referier- 
ten Studien kommen zu einem [statistisch] signifikanten Abschreckungseffekt der Todesstrafe. Nur eine Studie stellt diese Ergebnisse in Frage. Für einen Ökonomen ist das, was ich Ihnen sage, nicht überraschend: Wir erwarten, dass die Verbrecher ebenso wie die potentiellen Verbrecher auf die Drohung mit einer Sanktion reagieren, und die Todesstrafe ist die schwerste der Strafen, die wir anwenden“ (Rubin 2006: o. S.). Insgesamt, schreibt er, sei „die wissenschaftliche Literatur leicht zusammenzufassen".

Vor der gleichen Enquetekommission kommt Jeffrey Fagan, Professor of Law and Public Health an der Columbia Law School zu genau entgegengesetzten Schlussfolgerungen. Das Gutachten Fagans ist etwas länger, da er sich mehr über den Kontext jeder einzelnen Studie auslässt: Anstatt den gordischen Knoten zu zerschlagen, zieht Fagan es vor, ihn langsam aufzudröseln. Die Zusammenfassung gelingt gleichwohl in einem einzigen Absatz: „Die Studien [die sich für die Todesstrafe aussprechen] wimmeln von technischen und konzeptuellen Fehlern“: „fehlende Daten“, „ungetestete Kontrollvariablen“, die „Unmöglichkeit, die Ergebnisse auf der Grundlage der gleichen Daten zu replizieren“, und weitere Mängeln.

Man könnte nun den Eindruck gewinnen, dass die Uneinigkeiten sich auf eine Frage von Qualität und Quantität der herangezogenen Arbeiten reduzieren ließen. Aber sie werden der Kommission nicht nach diesem Schema vorgestellt. Paul Rubin äußert sich gegenüber der Kommission hinsichtlich einer Kritik seiner Arbeiten, die von Jeffrey Fagan herangezogen wurde, wie folgt: „Die Ergebnisse dieser Kritik ${ }^{4}$ sind nicht von kompetenten Forschern evaluiert worden. Die Stanford Law Review wird - wie alle rechtswissenschaftlichen Zeitschriften - von Studenten herausgegeben, die keine hinreichenden Kenntnisse in Ökonometrie besitzen" (ebd.). Jeder präsentiert also gegenüber der Politik ein Argument, das gleichzeitig quantitativ und qualitativ ist. Jedem Gutachten zufolge stützen die meisten Arbeiten den eigenen Standpunkt, und Arbeiten, die diesen Standpunkt kritisieren, sind von schlechter Qualität.

Zwei klassische Erklärungsansätze des Streits

Zwei Erklärungsansätze sind vorgeschlagen worden, um die Disparität der Aussagen zur abschreckenden Wirkung der Strafen und insbesondere der Todesstrafe auf den Begriff zu bringen. 
Der erste Erklärungsansatz geht von den Argumenten aus, die diesem Forschungsbereich eigen sind. Demnach variiert die abschreckende Wirkung der Todesstrafe nicht nur danach, welche Strafe und welches Verhalten untersucht würden, sondern wenigstens bei der Todesstrafe auch danach, welche Bundesstaaten betrachtet (Shepherd 2005), welche Art von Daten herangezogen (Yang/Lester 2005) werden sowie von weiteren Parametern wie den herangezogenen Kontrollvariablen (Dölling et al. 2009). Eben weil das Phänomen der abschreckenden Wirkung in sich selbst heterogen ist, bilden auch die veröffentlichten Arbeiten ein heterogenes Phänomen. Die wissenschaftliche Disparität reflektiere eine Disparität in der Wirklichkeit, und die Aufgabe der Wissenschaft bestehe darin, die Fälle zu finden, in denen die Strafe funktioniert, und diejenigen, in denen sie nicht funktioniert.

Der zweite Ansatz greift nicht bei der abschreckenden Wirkung von Strafen an, sondern bei der empirischen Forschung an sich. Man könnte hier die Argumente zusammenfassen, die die Seriosität der Studien, die Voreingenommenheiten und Prädispositionen der Autoren heranziehen, um die divergierenden Resultate zu erklären (Donohue/Wolfers 2006; Leamer 1982, McManus 1985). Der Blick, nicht das Betrachtete, erklärt die Disparität. Hier wird die „Ziffer“ als soziale Konstruktion verstanden, und das Interesse richtet sich darauf, wie unterschiedliche wissenschaftliche Unternehmungen unterschiedliche Ziffern über die gleiche Realität produzieren.

Der erste Erklärungsansatz ist in zahlreichen Berechnungen verwendet worden. Die wissenschaftliche Gemeinschaft verfügt über eine Reihe von Werkzeugen, die Disparitäten innerhalb einer gegebenen Forschungsliteratur zu quantifizieren; eines davon ist die Anfertigung von Meta-Analysen. Drei jüngere Arbeiten zum Abschreckungseffekt der Todesstrafe versuchen, die Disparität der Resultate im Wesentlichen auf „methodische Differenzen“ zurückzuführen (Dölling et al. 2009; Durlauf/Fu/Navarro 2013; Yang/Lester 2008), das heißt letztlich auf Unterschiede in den verwendeten Datensätzen und den Modellen. Wie in vielen anderen Bereichen auch leisten Meta-Analysen Vergleiche zwischen verschiedenen Arbeiten in Bezug auf die Datenarten, die herangezogen werden, oder die Art der Gesetzesübertretung, die in den Blick genommen wird. Wir erfahren hier, dass bei Studien über die abschreckende Wirkung des Strafsystems die Angabe der Effektstärke davon abhängt, ob sie sich über den Abschreckungseffekt durch die Härte der Strafe oder durch deren Wahrscheinlichkeit äußern, und dass Arbeiten sich dann 
eher zugunsten der Todesstrafe aussprechen, wenn Zeitreihen- statt Paneldaten verwendet werden. Mitunter ist es so, dass die Unterschiede „Sinn machen", und dementsprechend wird eine Erklärung zurechtgelegt: Wenn die negative Korrelation Strafe-Verbrechen bei Studien über Eigentumsdelikte größer ist als bei Gewaltdelikten, liegt dies zweifellos daran, dass es sich bei ersteren um rationalere Delikte handelt. Die veröffentlichten Studien werden als Fenster in eine bestimmte positive Wirklichkeit betrachtet, wobei die Rolle der Meta-Analyse darin besteht, die verschiedenen Realitäten miteinander zu vereinigen, d.h. ihre allgemeine Übereinstimmung mit einem Modell aufzuzeigen.

Anders als bei diesen Erklärungsansätzen ist die Suche nach Verzerrungen durch Merkmale der Autoren und Autorinnen theoretisch intensiv entwickelt worden, hat sich aber nicht wirklich in groß angelegten Quantifizierungsversuchen niedergeschlagen (zur Integration der Variable „Beruf des Autors“ in eine Metaanalyse siehe Gerritzen/Kirchgässner 2013). Allerdings lässt die Erklärung der Unterschiede in den Resultaten aus der angewandten Methode offen, warum die einzelnen Autoren die Gesamtheit des Feldes je anders angehen; soviel nämlich lässt sich der Lektüre der „Forschungsstände“ oder dem Anhören von Gutachten entnehmen. Anders gesagt: Es mag wohl sein, dass bestimmte Arten von Daten oder Modellen eher geeignet sind, Resultate zu liefern, die eine bestimmte These stützen können. Das erklärt aber nicht, warum nicht alle Autoren und Autorinnen die Gesamtheit der einschlägigen Literatur in der gleichen Weise verstehen.

Außerdem birgt der Versuch, sich mit einer Erklärung zufrieden zu geben, die rein auf Eigenheiten der Todesstrafe oder des Abschreckungseffekts von Strafen basiert, die Gefahr in sich, hinter dieser Frage ein tiefer liegendes, grundsätzlicheres Problem zu übersehen: Immerhin ist die Situation, in der wir die Forschung zur Todesstrafe vorfinden, nicht einzigartig, sondern wiederholt sich in vielen anderen Bereichen: Auch anderswo haben wir eine eher unverdauliche quantitativ-interdisziplinäre Literatur, die jeweils radikal entgegengesetzte Ergebnisse, Interpretationen und Expertisen liefert. Eine Literatur, die bestimmt ist von ihrer Diversität (die von Forschern und Forscherinnen hervorgebracht wird, die aus verschiedenen Disziplinen und verschiedenen Zirkeln kommen, in verschiedenen Kontexten arbeiten und fast nie zweimal die gleiche Methodik anwenden, was den Vergleich schwierig macht) und ihren Regelmäßigkeiten: Publikationsorte (referierte Zeitschrif- 
ten), starke Mathematisierung der Untersuchung, eine gewisse Undurchschaubarkeit der Herangehensweise (im Gegensatz zu einfachen Vergleichen von Kriminalitätsraten). Diese Akkumulation diversifizierter, undurchschaubarer und widersprüchlicher Literatur finden wir auch auf anderen Forschungsfeldern: Die Evaluierung des Rückfälligkeitsrisikos, insbesondere von Sexualstraftätern (wo jüngst der Versuch unternommen worden ist, zu nüchterneren Zahlen zurückzukehren, siehe Helmus et al. 2012), die Messung der Kriminogenität (oder ihrer Nichtexistenz) des Gefängnisses (Jonson 2010; Lipsey/Cullen 2007) oder auch der Einfluss von „Subkulturen“ auf die Kriminalität (Pratt/Cullen 2005) mögen als Beispiele dienen. Einige Felder, etwa die Korrelation zwischen der freien Zugänglichkeit zu Feuerwaffen und Tötungsdelikten, ähneln stark der Forschung zum Abschreckungseffekt der Todesstrafe: Wir finden die gleichen Modelle, die gleichen Debatten, mitunter sogar die gleichen Akteure (Donohue/Ayres 2009). Jedes Mal stehen sich die gleichen Grüppchen, Schulen, Netzwerke, ganze Disziplinen gegenüber und produzieren Zahlen, die angesichts des Umfangs der wissenschaftlichen Literatur kaum noch zusammenzufassen sind. Man ist also darauf verwiesen, sich die Frage zu stellen, ob sich diese Disparitäten jedes Mal durch konkrete kontextuelle Unterschiede, durch die diskutierten Fragenkomplexe selbst erklären lassen, oder ob wir es mit einem allgemeineren Problem zu tun haben, das in der Perspektivendiversität der Forscher und Forscherinnen gründet, die diese Arbeiten anfertigen.

Damit ergibt sich die Notwendigkeit, bewährte quantitative Methoden einzusetzen, um die Erklärungskraft dieser zweiten Perspektive zu quantifizieren und so auf der gleichen Basis aufzubauen wie positivistische Erklärungen und um auf diesem Wege zu überprüfen, ob der Autor oder die Autorin nicht ebenso wie die „Wirklichkeit“ ein Faktor ist, der zum Verständnis der Disparitäten in den Forschungsergebnissen beiträgt.

\section{Die Nicht-Gaußsche Verteilung der Resultate}

Es entspricht den Erwartungen, wenn Studien über den gleichen Gegenstand aber in unterschiedlichen Kontexten und unter Verwendung unterschiedlicher Methoden und Daten zu unterschiedlichen Resultaten kommen. Wenn aber diese methodologischen Unterschiede nur die Frucht des Zufalls sind, dann könnte man auch erwarten, dass diese Resultate ungefähr gleichmäßig um 
einen Mittelwert gruppiert sind, der gleichzeitig der Median wäre. Forschungsfelder, deren Resultate nicht „normal“ um einen Mittelwert verteilt sind, leiden möglicherweise unter einer verzerrenden Veröffentlichungspraxis (Egger et al. 1997). Das heißt, dass bestimmte Resultate möglicherweise in den einzelnen Zeitschriften unproportional über- oder unterrepräsentiert sind.

Yang und Lester haben eine Bibliographie aller 100 zwischen 1970 und 2008 erschienenen quantitativen Arbeiten zur Todesstrafe vorgelegt, die die Arbeiten in fünf Kategorien einordnet (I. Zeitserien II. Geographische Daten III. Paneldaten IV. Bekanntgabe der Hinrichtung V. Einfluss einer einzelnen Hinrichtung auf Tötungsdelikte in den darauffolgenden Tagen). 85 Aufsätze (die Kategorien I, II und III) beschäftigen sich eigentlich mit der gleichen Frage, nämlich mit der statistischen Korrelation zwischen der Zahl der Hinrichtungen und der Zahl der Tötungsdelikte; was sich hier unterscheidet, ist also nicht die Forschungsfrage, sondern die Art der verwendeten Daten (zeitlich, räumlich oder gemischt).

Der Mittelwert, den Yang und Lester für die Gesamtheit der Ergebnisse angeben, beträgt $-0,126$; sie interpretieren diesen Wert als „Ergebnis, das die These, es gebe einen abschreckenden Effekt der Todesstrafe, unterstützt“ (Yang/Lester 2008: 453). Wären nun die mittleren Wirkungsgrade in diesen Aufsätzen normal um den Wert von -0,126 verteilt, wäre zu erwarten, dass die Ergebnisse der Studien sich in einer Gaußschen Glockenkurve um diesen Wert herum anordnen würden.

Schaubild 1 belegt allerdings, dass dies nicht der Fall ist. Die Grafik zeigt für jede Kategorie die Verteilung der Resultate für jede Effektstärke (+/$0,025)$. Jede Graustufe repräsentiert einen der drei oben erwähnten Datentypen (Grafik 3, die weiter unten besprochen wird, diskutiert die Verteilung der Resultate für jeden Datentyp). Man kann nun über diese Verteilung eine Gaußsche Kurve als Darstellung der Normalverteilung für einen Mittelwert von $-0,125$ (den von Yang und Lester berechneten Mittelwert) und eine Standardabweichung von $-0,1$ legen. Es ist ohne weiteres zu sehen, dass eine solche Kurve in keiner Weise deckungsgleich ist mit der Art, in der die Ergebnisse verteilt sind. 
Schaubild 1: Abweichungen der Resultate von Forschungen über die Korrelation zwischen Hinrichtungen und Tötungen von der Erwartungen einer Gaußschen Verteilung beiderseits des Mittelwertes

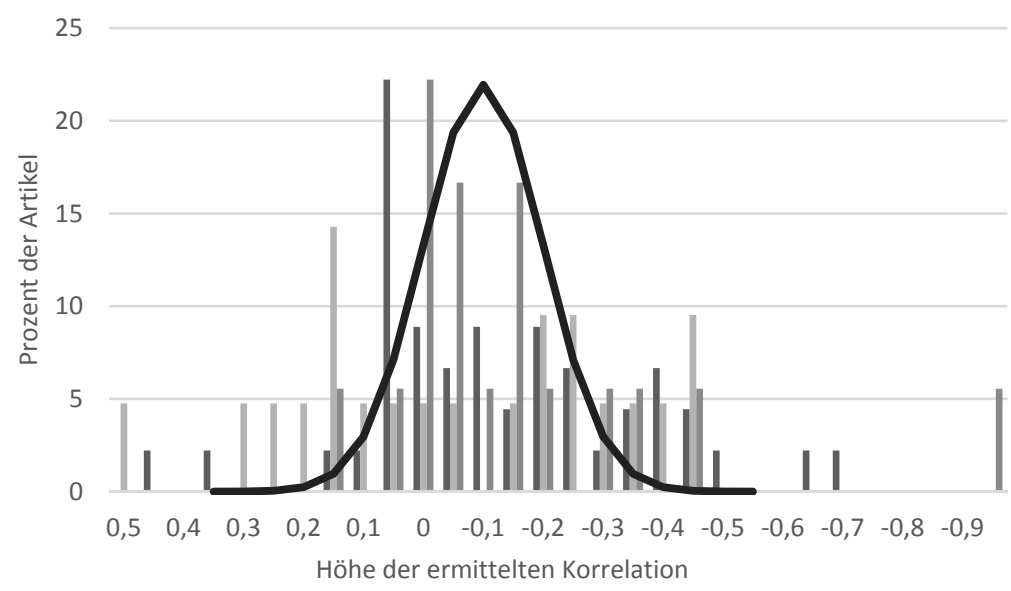

Anhand von Grafik 2 lässt sich aufzeigen, wie die Resultate tatsächlich verteilt sind: Die Mehrheit der Veröffentlichungen gravitiert „,normal“ um 0,025 (d.h. fast einen Null-Effekt der Todesstrafe) mit einer Standardabweichung von -0,1, und zwei Gruppen von eher minoritären Forschungen finden sich in Normalverteilungen um -0,2 und links um -0,45. Diese minoritären Studien sprechen sich vehement für die Todesstrafe aus, scheinen aber von einer anderen wissenschaftlichen Normalität zu berichten. Ihre Existenz und der extreme Charakter ihrer Resultate bewirkt, dass das allgemeine Mittel nach unten gezogen und den Eindruck erweckt wird, dass das gesamte Feld eine abschreckende Wirkung der Todesstrafe belegt, während das häufigste Ergebnis doch Null oder fast Null lautet. Der resultierende Mittelwert beschreibt damit nicht eine positive Realität, wie sie eine vereinheitlichte Untersuchung normalerweise erbringen würde, sondern den Mittelwert dreier wissenschaftlicher Normalverteilungen. Nun ist dieser Mittelwert sicherlich aufschlussreich für die soziale Produktion des Wissens über die Todesstrafe; er hat jedoch keinen besonderen Aussagewert über das Phänomen der Todesstrafe. 
Schaubild 2: Die Mehrheitsforschung und zwei minoritäre Forschungen

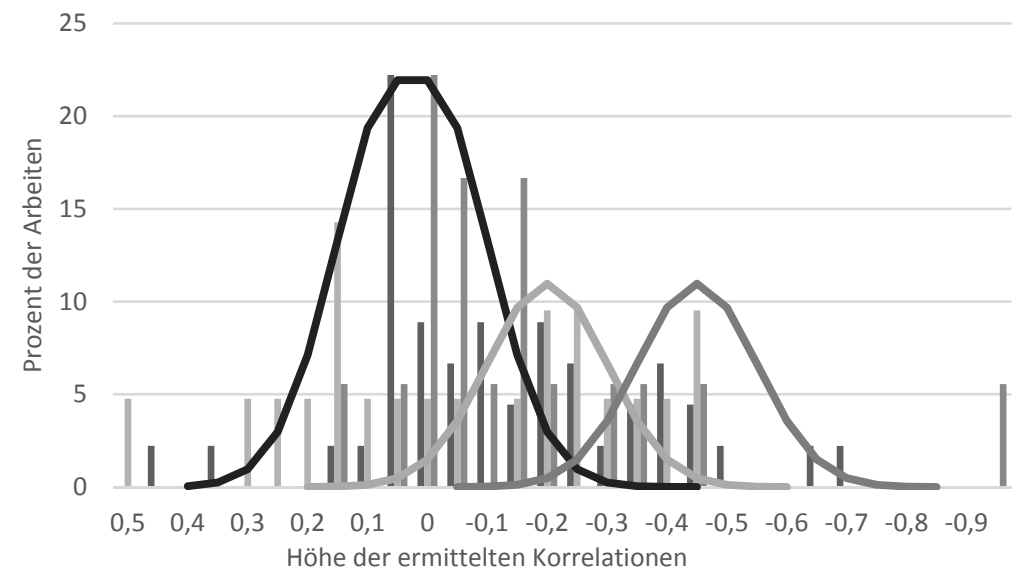

Diese Gliederung in eine Mehrheitsforschung, die zum Schluss kommt, dass der Effekt der Todesstrafe auf Tötungsdelikte gegen Null tendiert, und einer Minderheitsforschung, die zum entgegengesetzten Schluss kommt, hängt nicht von den verwendeten Daten ab - obwohl gerade dies am häufigsten als Erklärung für die Disparität der Resultate herangezogen wird (Yang/Lester 2008). Schaubild 3 zeigt, dass vier der fünf verwendeten Datentypen exakt die gleiche Verteilung zeigen: $\mathrm{Ob}$ das Interesse auf der Korrelation Hinrichtungen-Tötungsdelikte in der Zeit (A), im Raum (B), in Paneldaten (C) gerichtet ist, und selbst wenn eine Verbindung zwischen der Bekanntmachung einer Hinrichtung und Tötungsdelikten gesucht wird (D), was nicht einmal die gleiche Fragestellung ist, finden wir immer wieder das gleiche Phänomen: Die Mehrheit der Studien gravitiert um Null, während eine Minderheit zu extremen Ergebnissen kommt. Die einzige Untergruppe, wo die Verteilung der Resultate abweicht, besteht aus einigen wenigen Arbeiten über den Einfluss einer einzigen Hinrichtung auf die Tötungsdelikte in den darauffolgenden Wochen. Es handelt sich um fünf Arbeiten, die von nur drei verschiedenen Gruppen produziert wurden. Es ist also kaum möglich, sie in verallgemeinerbarer Form zu analysieren oder mit den anderen zu vergleichen. 
26. Jahrgang 2015, Heft 1

Sacha Raoult

Schaubild 3: Zahl der Arbeiten je Effektstärke in vier Typen von Arbeiten

A.

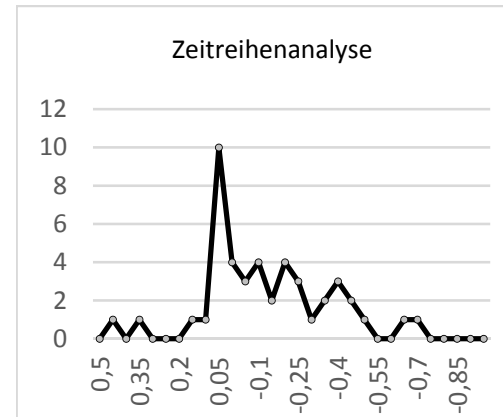

C.

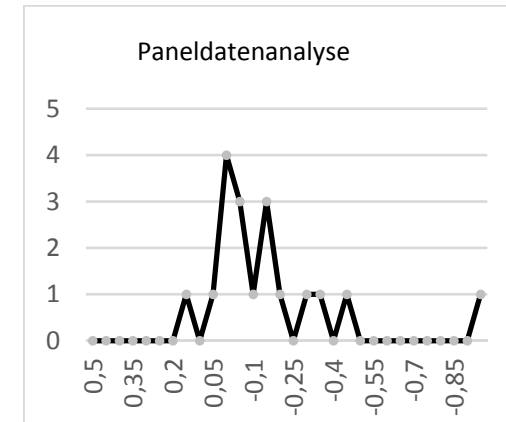

B.

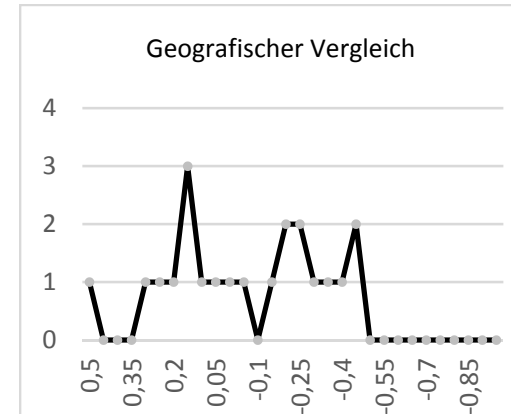

D.

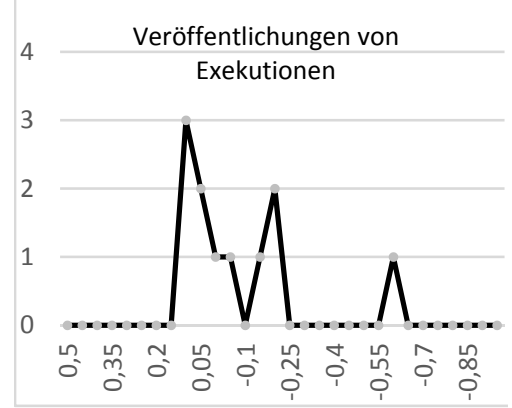

Diese Nicht-Gaußsche Verteilung führt uns zu der Frage, ob es nicht sinnvoller und ergiebiger wäre, anstelle der Suche nach Verzerrungen in den Daten die Frage eines Bias bei den Autoren zu beantworten und zu untersuchen, ob es nicht immer die gleichen Autoren und Autorinnen sind, die dazu tendieren, die gleichen Resultate hervorzubringen.

\subsection{Die Identifizierung von Trends bei den Autoren}

Yang und Lester (2008: 453) liefern für jede Publikation zwei Angaben:

- Eine mittlere Effektstärke, die sie anhand der im Originalaufsatz genannten Daten berechnet haben; 
- die Schlussfolgerungen des Autors, wobei es mitunter zu einer Divergenz zwischen diesen Schlussfolgerungen und der berechneten Effektstärke kommt.

So kann ein Autor beispielsweise einen Effekt finden, der für (negativ) oder gegen (positiv) die Todesstrafe spricht, dann aber auf die geringe Stichhaltigkeit dieser Resultate hinweisen. Das repräsentativste Beispiel für einen solchen Widerspruch ist zweifellos die Schlussfolgerung Edward Leamers nach einer negativen Effektstärke: „Meinem Gefühl nach sind diese Daten über einen Abschreckungseffekt der Todesstrafe nicht stichhaltig genug, um ihnen Vertrauen zu schenken“ (1983: 42). Yang und Lester notieren daher „gemischte Schlussfolgerungen“ in ihrer Datenbasis neben der von Edward Leamer entdeckten Effektstärke.

Für unsere Untersuchung haben wir diese Bibliographie neu durchgearbeitet, wobei wir jeder Publikation zwei Ziffern zugeordnet haben:

- eine Bruttoeffektstärke, die von den Autoren selbst berechnet wurde

- eine korrigierte Effektstärke, d.h. eine Ziffer, in die die Schlussfolgerungen des Autors eingegangen sind.

Zur Kodierung der „korrigierten Effektstärke“ haben wir eine negative Effektstärke eingesetzt, wenn Yang und Lester notiert haben: „Schließt auf einen Abschreckungseffekt" und eine positive Effektstärke, wenn sie verzeichnen: „Schließt auf einen brutalisierenden Effekt". Wo es dagegen einen Widerspruch zwischen der Bruttoeffektstärke und den Schlussfolgerungen gibt, führt dies zu einer „Korrektur“ der Zahlen. Wir haben daher ,gemischte Schlussfolgerungen" und "schließt auf einen Nulleffekt" in eine Effektstärke der Größe Null übersetzt. Der Sinn der zwei Serien von Ziffern für jede Publikation besteht darin, zeigen zu können, dass alle Trends, die wir in unserer Studie entdeckt haben, für die korrigierten Effektstärken noch ausgeprägter sind als für die in den Arbeiten berichteten Bruttoeffektstärken. Das heißt, dass die subjektive Einschätzung seitens Autoren noch weitaus vorhersehbarer ist als die Bruttoeffektstärken, die sie finden.

Wer produziert die Zahlen über die Todesstrafe? Wir können innerhalb der 85 untersuchten Studien eine Untergruppe von Veröffentlichungen ausmachen, die von Autoren produziert wurden, die man „Spezialisten für die Korrelation Hinrichtungen-Tötungsdelikte" nennen könnte: Forschergruppen, die dieser Frage mehr als ein Papier gewidmet haben. Diese Forschergruppen 
haben wir möglichst weit definiert, um eine aussagekräftige Zahl an Daten zu gewinnen, nämlich als Netzwerk von Personen, die mindestens einen Artikel auf unserer Liste gemeinsam als Koautoren verfasst haben. Mit dieser Definition zählen wir 28 Spezialisten für die Korrelation Hinrichtungen-Tötungsdelikte zwischen 1970 und 2008 in 16 Forschergruppen, die für 64 der 85 veröffentlichten Aufsätze verantwortlich zeichnen - also für einen wesentlichen Bereich des Feldes. Nachdem wir das Feld in dieser Weise eingegrenzt haben, können wir nun die Korrelation zwischen den Ergebnissen eines Papiers und dem vorherigen Ergebnis der gleichen Gruppe bestimmen.

Die erste Art, in der wir dieses Verhältnis messen wollen, besteht im Vergleich der Ergebnisse einer von einer bestimmten Gruppe hervorgebrachten Publikation $p$ mit der folgenden Publikation $p+1$. Wenn die Bruttoeffektstärken der Veröffentlichungen $p$ und $p+1$ der gleichen Gruppe völlig voneinander unabhängig wären, müssten sich die Punkte mit den Koordinaten $(p, p+1)$ zufällig über die Grafik 4 verteilen. Dies ist jedoch nicht der Fall; man sieht vielmehr einen leichten Trend dahin, dass Publikationen mit einem bestimmten Ergebnis weitere mit ähnlichem Ergebnis folgen (dies ist der Trend, der von der Geraden dargestellt wird). Im unteren linken Viertel der Grafik, das heißt für die negativen Resultate (die für die Todesstrafe sprechen), denen ein weiteres negatives Resultat folgt, sehen wir eine Gerade; diese indiziert aufeinanderfolgende relativ ähnliche Ergebnisse (auf extreme Ergebnisse folgen extreme Ergebnisse, gemäßigten folgen gemäßigte Resultate).

In Schaubild 5 wird die gleiche Perspektive auf die korrigierten Effektstärken angewendet; der Trend ist hier noch deutlicher ausgeprägt. Das heißt: die Tendenz der Autoren, zwei Mal zu den gleichen Schlussfolgerungen zu gelangen, ist noch größer als die, zwei Mal hintereinander die gleichen Zahlen anzugeben. 
Empirische Sozialforschung und ihre Macher

26. Jahrgang 2015, Heft 1

Schaubild 4: Tendenz einer Forschergruppe, zuvor in der Publikation $p$ erhaltene Resultate in der folgenden Publikation $(p+1)$ zu bestätigen (Bruttoeffektstärken)

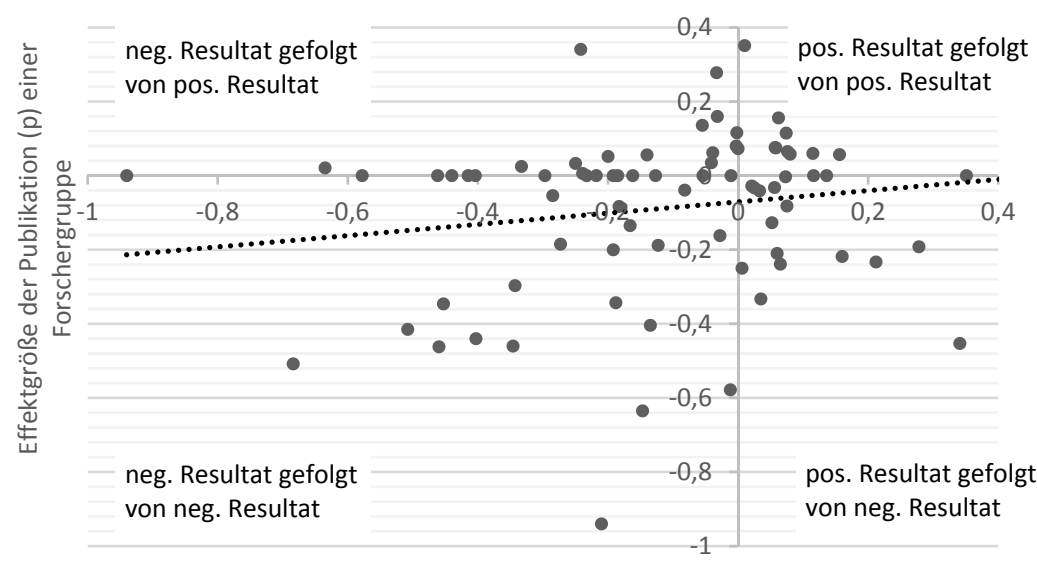

Effektgröße der folgenden Publikation $(p+1)$ derselben Forschergruppe

Schaubild 5: Tendenz einer Forschergruppe, zuvor in der Publikation p erhaltene Resultate in der folgenden Publikation $(p+1)$ zu bestätigen (korrigierte Effektstärken)

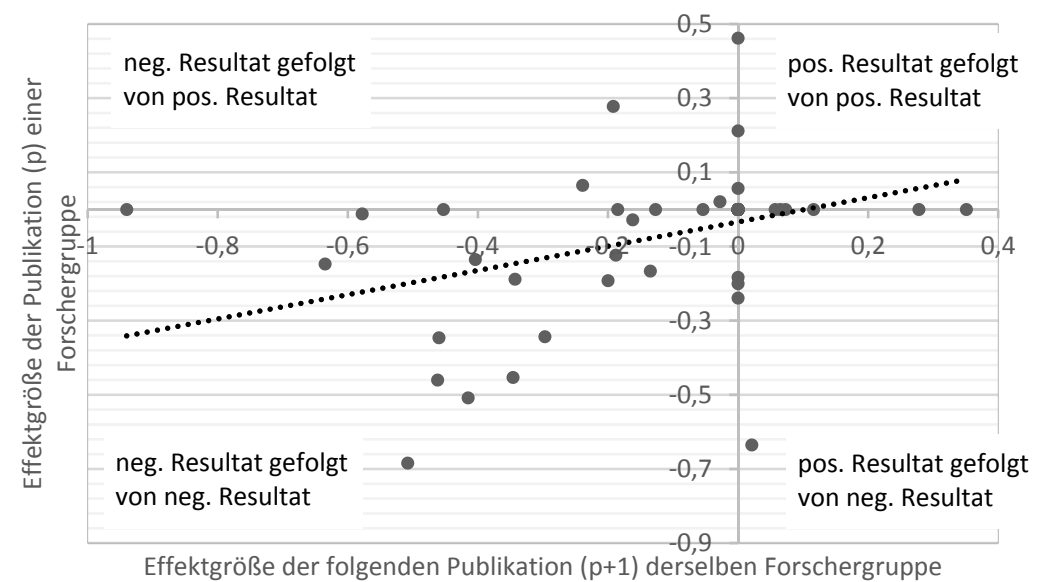

Effektgröße der folgenden Publikation $(p+1)$ derselben Forschergruppe 
Der zweite Vergleich, den wir anstellen werden, ist der der Mittelwerte der Veröffentlichungsresultate nach den Kategorien verwendete Daten und Autorennetzwerke. Dieser Vergleich wird in Grafik 6 dargestellt. Es zeigt sich, dass die Kenntnis des ersten Ergebnisses, das eine Forschergruppe in ihrer Karriere erhalten hat, ein besserer Prädiktor für ihre nachfolgenden Studien ist als das Wissen darüber, ob zeitliche, räumliche oder Paneldaten verwendet wurden - obwohl dieses Merkmal immer wieder als der wichtigste „BiasFaktor" angegeben wird, wenn es um Einflüsse auf Studien über die Todesstrafe geht.

\section{Schaubild 6: $\quad$ Mittelwert der Bruttoeffektstärken der Publikationen nach den verwendeten Daten (Spalten 1 bis 3) und Mittelwerte der Bruttoeffektstärken der folgenden Aufsätze einer Gruppe nach dem ersten von dieser Gruppe veröffentlichten Resultat (Spalten 4 bis 8)}

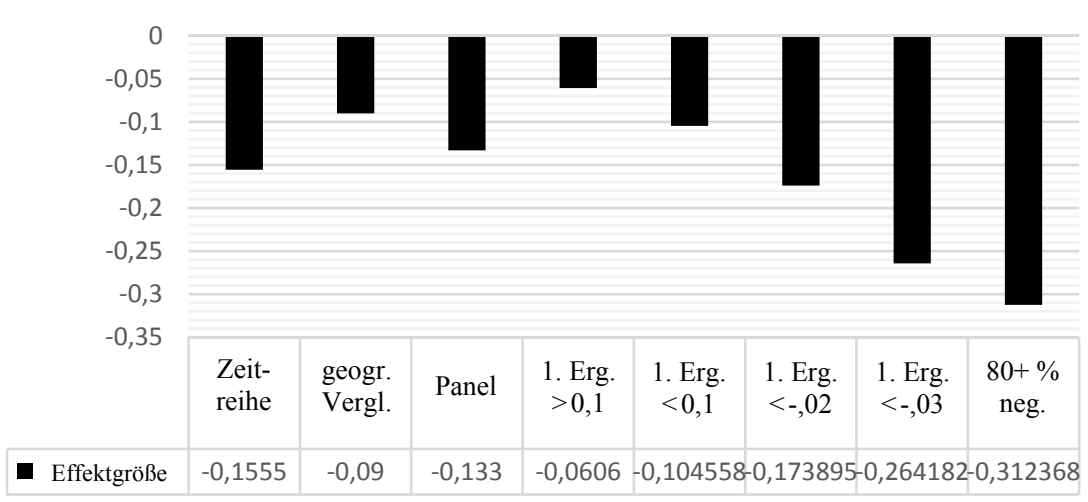

Wir haben es also mit zwei Elementen zu tun. Zunächst dem Umstand, dass die Forschungsergebnisse über die Korrelation zwischen der Zahl der Hinrichtungen und der Zahl der Tötungsdelikte unabhängig von den verwendeten Datentypen immer gleich verteilt sind: Eine Mehrheit der Studien findet keine Korrelation, und eine Minderheit von Studien findet große Korrelationen. Das zweite Element, das wir entdeckt haben, ist die Tendenz der Forscher, frühere eigene Resultate zu bestätigen. Wir können also die Forschergruppen gemäß ihren negativen Effektstärken aufteilen (Tabelle 1). Während nun der Gesamtanteil negativer Resultate bei 37 Prozent liegt, haben vier 
Empirische Sozialforschung und ihre Macher

26. Jahrgang 2015, Heft 1

Gruppen 100 Prozent negative Ergebnisse, eine fünfte 80 Prozent. Die Differenz zwischen dieser Verteilung und einer gleichmäßigen Verteilung ist so groß, dass eine Erklärung notwendig wird $\left(\mathrm{chi}^{2}=0,028\right)$.

Tabelle 1: Autorengruppen, die 2008 mindestens zwei quantitative Studien zur Korrelation zwischen Hinrichtungen und Gewaltverbrechen veröffentlicht haben

\begin{tabular}{l|c|c|c} 
Forschergruppe & $\begin{array}{c}\text { Zahl der } \\
\text { Studien }\end{array}$ & $\begin{array}{c}\text { korrigierte } \\
\text { negative } \\
\text { Effektstärken }\end{array}$ & $\begin{array}{c}\text { Hypergeometrische } \\
\text { Wahrscheinlichkeit }\end{array}$ \\
\hline Bechdolt & 2 & $0 \%$ & $39 \%$ \\
Bowers/Pierce & 2 & $0 \%$ & $39 \%$ \\
Decker/Kohfeld & 5 & $0 \%$ & $8.6 \%$ \\
Fox/Radelet & 2 & $0 \%$ & $39 \%$ \\
McAllee/Veall & 2 & $0 \%$ & $39 \%$ \\
Passel/Taylor & 2 & $0 \%$ & $39 \%$ \\
Peterson/Bailey & 20 & $10 \%$ & $0.2 \%$ \\
Yang/Lester & 4 & $25 \%$ & $37 \%$ \\
Avio & 2 & $50 \%$ & $47.6 \%$ \\
Leamer & 2 & $50 \%$ & $47.6 \%$ \\
Zimmermann & 2 & $50 \%$ & $47.6 \%$ \\
Dezhbakhsh/Rubin/Shepherd & 5 & $80 \%$ & $5.6 \% *$ \\
Clonninger/Blumm/Marchesini & 4 & $100 \%$ & $1.7 \%$ \\
Ehrlich/Liu & 4 & $100 \%$ & $1.7 \%$ \\
Layson & 3 & $100 \%$ & $4.9 \%$ \\
Yunker & 3 & $100 \%$ & $4.9 \%$ \\
\hline
\end{tabular}

* 5,6 Prozent ist die Wahrscheinlichkeit, dass eine Gruppe, die 5 Untersuchungen angestellt hat, genau 4 negative Resultate erzielt hat. Die Wahrscheinlichkeit, dass diese Gruppe mehr als 4 negative Resultate erzielt hat, beträgt 6,1 Prozent. Zu den Quellen siehe Anmerkung 2.

In Tabelle 1 wurde außerdem die hypergeometrische Wahrscheinlichkeit dafür berechnet, dass eine Gruppe exakt das Verhältnis negativer/positiver Resultate erhält, die sie erhalten hat, wobei vom offensichtlich fiktiven Prinzip ausgegangen wurde, dass am Ende 24 von 64 Resultaten negativ sein müssen. Dabei zeigt sich eine Schwelle ab 80 Prozent negativer Resultate. Wenn also alle Forscher und Forscherinnen die gleiche Sache in gleicher Weise betrachten würden, läge die Wahrscheinlichkeit dafür, dass eine bestimmte 
Gruppe so viele zugunsten der Todesstrafe sprechende Resultate zusammentrüge, sehr niedrig, nämlich bei 6,1, 4,9 und 1,7 Prozent. Mit anderen Worten: Es ist sehr wahrscheinlich, dass die Art, in der die Autoren schauen, erklärt, wieso sie so viele negative Korrelationen finden (wobei Sensibilitätsanalysen zeigen, dass man auf der Grundlage der gleichen Daten durchaus andere Dinge sehen kann, siehe Yang 1998; Donohue/Wolfers 2006; Kirchgässner 2011).

\subsection{Die Profile der Forscher im Verhältnis zu ihren Resultaten}

Zwei Gruppen von Forschern. Die Forscher und Forscherinnen lassen sich in zwei Gruppen (A und B) aufteilen, abhängig davon, wo sie sich in Bezug auf die Schwelle von 80 Prozent negativer Resultate befinden. Ab dieser Schwelle fällt die hypergeometrische Wahrscheinlichkeit, die eigenen Resultate unter Verwendung der gleichen Herangehensweise wie die anderen Forscher zufällig erreicht zu haben, drastisch ab, und zwar auf jeden Fall unter 6 Prozent. Vor dieser Schwelle weisen zwei Gruppen eine Wahrscheinlichkeit von weniger als 9 Prozent auf, während sie bei den meisten um 30-40 Prozent beträgt.

Dieser Schwellenwert ist auch deshalb interessant, weil die einzige Gruppe in dieser Kategorie A, die positive Ergebnisse erzielt hat, im Übrigen auch der Todesstrafe sehr positiv gegenübersteht. Tatsächlich ist die Interpretation der Schlussfolgerungen von Yang und Lester über die Studie von Joanna Shepherd, die sie im Jahre 2005 alleine publiziert hat und das einzige nicht negative Resultat in dieser Kategorie ist, unvollständig. Shepherd berechnet die Korrelation zwischen Hinrichtungen und Tötungsdelikten in mehreren amerikanischen Bundesstaaten. Bildet man den Mittelwert aller Resultate, dann findet man eine leichte positive Effektstärke, allerdings interpretiert die Autorin selbst diese Ziffern in folgender Weise: „Meine Ergebnisse ziehen wichtige politische Schlussfolgerungen nach sich: Um einen Abschreckungseffekt zu erzielen, muss der Staat eine bestimmte Anzahl von Personen hinrichten“ (Shepherd 2005a: 1). Während Yang und Lester schreiben, dass „die Autorin einen brutalisierenden Effekt feststellt", sehen wir, dass die gleichen Ergebnisse von der Autorin so interpretiert werden, dass sie - wenn auch mit Nuancen - einen Abschreckungseffekt der Todesstrafe bestätigen. Betrachten wir nun noch den popularisierenden Aufsatz, den Joanna Shepherd im Chris- 
Empirische Sozialforschung und ihre Macher 26. Jahrgang 2015, Heft 1

tian Science Monitor veröffentlicht hat. Dort teilt sie uns mit: „Meine intuitive Erklärung [dieser positiven Resultate] ist, dass man einen abschreckenden Effekt nur dann feststellen kann, wenn ein Staat hinreichend viele Leute hin-

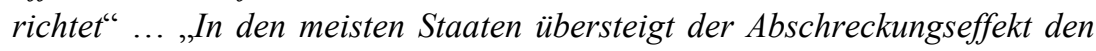
brutalisierenden Effekt, wenn die Zahl der Hinrichtungen eine bestimmte Schwelle überschreitet" (Shepherd 2005b: o.S.). Es gibt also offensichtlich eine Gruppe von Forschern und Forscherinnen, die systematisch Resultate zugunsten der Todesstrafe erhält, mit der einzigen halben Ausnahme der zitierten Autorin.

\section{Unterschied zwischen den beiden Gruppen}

Eine der immer wiederkehrenden Hypothesen über die Disparität der Resultate hinsichtlich der Todesstrafe lautet, dass Studien zugunsten der Todesstrafe von Ökonomen produziert werden, während kritische Studien von Soziologen und Soziologinnen stammen. Es gibt Ökonomen und Soziologen, die diese Opposition ihrerseits vertreten (Goertzel/Goerzel 2008; Rubin 2006; Gerritzen/Kirchgässner 2013). Sie ist aber nur teilweise richtig. Tatsächlich stehen sich in unseren beiden Kategorien nicht zwei homogene Gruppen („Ökonomie“ und „Soziologie“) gegenüber; sie umfassen vielmehr

A. eine homogene Gruppe amerikanischer Ökonomen von wenig angesehenen Universitäten;

B. eine heterogene Gruppe von Soziologen, europäischen Ökonomen und amerikanischen Ökonomen von Universitäten hohen Ansehens.

Wir haben, um diese Profile zu erstellen, alle zugänglichen Angaben aus den Lebensläufen der 28 Autoren und Autorinnen entnommen. Manche dieser Angaben, die sicherlich für eine Untersuchung der Voreingenommenheit der Autoren von besonderem Interesse gewesen wären (die Quelle der Finanzierung von Studien, verschiedene Indikatoren für politische oder ideologische Positionen) waren sehr selten und erlauben es nicht, auf der Grundlage einer so kleinen Stichprobe zu verlässlichen Aussagen zu kommen. Gleichwohl haben sich schon bei den Punkten, die systematisch erfasst werden konnten, sehr deutliche Unterschiede ergeben.

In der Gruppe A, das heißt oberhalb der Schwelle von 80 Prozent Resultaten zugunsten der Todesstrafe, ist Joanna Shepherd die einzige Frau, die einzige, die ein nicht negatives Resultat veröffentlicht hat und die einzige, die 
eine Stellung als Juristin bekleidet (tatsächlich ist Joanna Shepherd gleichzeitig Juristin und Ökonomin, da ihre Stelle an zwei Beschäftigungen als Ökonomin und eine Abschlussarbeit in „Law and Economics“ anschließt; ihre Qualifikation auf der Seite der Fakultät ist ebenfalls „Law and Economics“, und sie publiziert regelmäßig in ökonomischen Zeitschriften zusammen mit Wirtschaftswissenschaftlern). Sie ist also in mehrerlei Hinsicht eine Ausnahmeerscheinung innerhalb ihrer Gruppe, auch wenn sie abgesehen von diesen Punkten einen sehr ähnlichen Werdegang durchlaufen hat wie die übrigen Angehörigen dieser Kategorie. Von ihr abgesehen umfasst die Gruppe A (10 Forscher) zu 100 Prozent männliche Ökonomen (Beschäftigung in „Economic“ oder „Finance“) aus den Vereinigten Staaten. Im Gegensatz dazu enthält Gruppe B (18 Forscher) unter denjenigen, deren Position bekannt ist, 50 Prozent Ökonomen, von denen 17 Prozent außerhalb der Vereinigten Staaten (nämlich in Kanada oder Europa) beschäftigt sind.

Andere Bestandteile der Lebensläufe unterscheiden sich in einer Weise, dass der Gesamteindruck zweier sehr unterschiedlicher Gruppen entsteht.

Zunächst einmal ist das am häufigsten gelehrte Fach in der Gruppe A die Mikroökonomie, d.h. die Modellierung rationaler Akteure, während die am häufigsten gelehrte Materie bei den Angehörigen der Gruppe B Crime and Punishment ist, was sich als Kriminologie oder Studium des Strafsystems übersetzen ließe.

Dann sind die Forschungsschwerpunkte in den beiden Gruppen sehr unterschiedlich. Die Lebensläufe beginnen sehr oft mit einem Absatz oder einer kurzen Liste, die das oder die Interessen des Autors präsentiert - sei es im Kopf des Lebenslaufs oder auf der Autorenwebseite neben dem Link zum Download des Lebenslaufs. Abgesehen von den zwei bis fünf Veröffentlichungen zur Todesstrafe zeigen sich die Autoren hier als Spezialisten für „Regionalökonomie“, „Chinesische Entwicklung“, „Pharmazeutik“ oder die „Verwendung neuronaler Netze für Kreditentscheidungen“. Dagegen finden wir in der Gruppe B Forscher, die als Interessengebiete neben der Todesstrafe „Feuerwaffen“, „Gangs“, „multiple Morde“, „Gewalt", „Jugenddelinquenz“ angeben. In der Gruppe A sind die Arbeiten zur Todesstrafe fast immer Fortsetzungen von Forschungen zum rationalen Konsumenten. In der Gruppe B sind sie entweder Fortsetzungen von Untersuchungen zum Verbrechen und zum Strafsystem oder methodologische Übungen, die die Fragwürdigkeit bestimmter ökonometrischer Arbeiten aufzeigen sollen. 
Die Veröffentlichungen unterscheiden sich gleichermaßen zwischen den Gruppen. Werden abgesehen von der Todesstrafe weitere juristische oder Strafinstitutionen untersucht, dann geht es bei den Angehörigen der Gruppe $\mathrm{A}$ in gleicher Weise um Wirksamkeit und Abschreckungseffekt - insbesondere bei den Feuerwaffen. Rationalität und die individuelle Reaktion auf Normenwandel sind das einzige, was die Angehörigen der Gruppe A an der Todesstrafe interessiert; von ihnen gibt es keine Arbeiten über Ungleichheit und Ungerechtigkeit in der Funktionsweise des Strafsystems, während diese einen Hauptgegenstand der Gruppe B bilden. Dieses mangelnde Interesse an Ungleichheiten ist überraschend: Da die Todesstrafe in sehr ungleicher Weise angewandt wird, sollte man annehmen, dass ein Forscher, der sich für den Abschreckungseffekte interessiert, sich auch die Frage stellt, welchen Einfluss die ungleiche Anwendung auf die Kriminalität haben mag.

Eine Information, die am häufigsten angegeben ist, und von der man erstaunt feststellt, dass sie ein sehr guter Prädiktor für die Ergebnisse einer Arbeit zur Todesstrafe ist, ist das Ranking der Universität der letzten Beschäftigung des Autors. Nun werden die Universitäten in den USA nach verschiedenen Systemen klassiert, die in der Regel zueinander passende Resultate liefern; wir verwenden hier das Ranking der Times Higher Education von 2013-2014. ${ }^{5}$ Harvard, Yale, das MIT, die Universität von Chicago, Stanford werden hoch eingestuft. Die Western Illinois University beispielsweise ist nicht erwähnt, die Emory University befindet sich im mittleren Feld. Das Ranking erfolgt nach einer Reihe von Kriterien, die die Times ausgewählt hat: Strenge der Aufnahmekriterien für Studierende, Budget je Studierendem, wissenschaftliche Produktion des Personals. Es ist an dieser Stelle nicht erforderlich, die Stichhaltigkeit dieser Kriterien zur Unterscheidung "guter“ von „schlechten“ Universitäten zu beurteilen, denn es unterliegt keinem Zweifel, dass diese Klassierung in den Vereinigten Staaten weitgehend diejenigen Universitäten angibt, die sozial als angesehen oder nicht betrachtet werden, und zwar sowohl in der akademischen Welt als auch unter den Studierenden. Nun ist es zunächst einmal sehr überraschend, dass dieses Ranking stark mit den Resultaten korreliert, die von den dort jeweils beschäftigten Forschern zur Todesstrafe hervorgebracht werden.

Diese Korrelation verstärkt sich noch drastisch, wenn man nur die Forschergruppen betrachtet, die ausschließlich aus Ökonomen bestehen („Economics“, „Finance“ und „Law and Economics“); es ergibt sich ein sehr hoher 
26. Jahrgang 2015, Heft 1

Sacha Raoult

Korrelationskoeffizient $\mathrm{R}^{2}$ von 0,55 . Dieser Befund wird in den Grafiken 7 und 8 dargestellt.

Schaubild 7: $\quad$ Korrelation zwischen dem Rang der Universität einer Gruppe und dem Prozentsatz negativer Resultate $\left(R^{2}=0,18\right)$

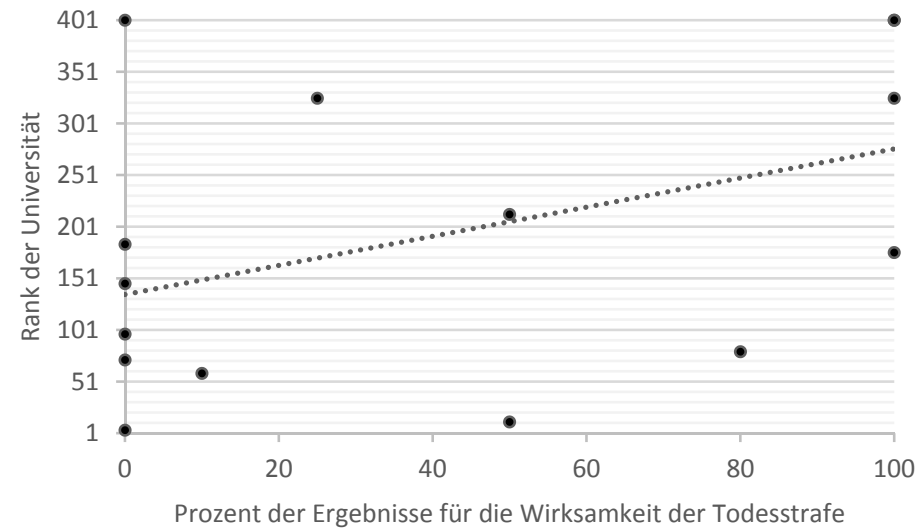

Schaubild 8: $\quad$ Korrelation zwischen dem Rang der Universität einer Gruppe von Ökonomen und dem Prozentsatz negativer Resultate $\left(R^{2}=0,55\right)$

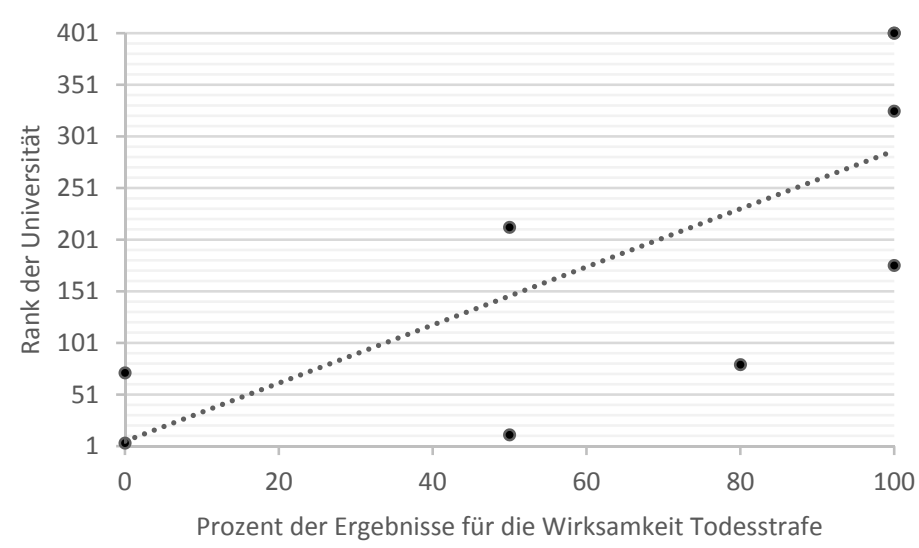


Tabelle 2 ermöglicht es, sich eine genauere Vorstellung von diesen Untergruppen zu machen, indem insbesondere der wichtige Prädiktor Ranking der Universität der letzten Beschäftigung verglichen wird mit einem weiteren Kriterium, nämlich dem Ranking der Universität, an der jeder Autor seinen Abschluss gemacht hat, wobei letzteres nicht als Prädiktor taugt. Tatsächlich gibt es keine sehr großen Unterschiede hinsichtlich des Niveaus der „Ausgangsuniversitäten“ der Forschenden: Acht der zehn Ökonomen in Gruppe A haben ihren Abschuss in einer der 200 bestplatzierten Universitäten gemacht, ebenso sechs von acht Ökonomen der Gruppe B sowie 12 von 18 Forschern in dieser Gruppe insgesamt. Dagegen gibt es enorme Unterschiede im Niveau der „Ankunftsuniversitäten“. Die Beschäftigung von drei Ökonomen der Gruppe B in den Top 50 (tatsächlich sogar in den Top 15), während sich kein Ökonom der Gruppe A dort wiederfindet, ist statistisch signifikant (Test von Student). Gleichwohl ist dieses Ergebnis aufgrund der kleinen Stichprobe nicht repräsentativ. ${ }^{6}$

Der Aufstieg oder Abstieg eines Forschers oder einer Forscherin zwischen der Ausgangs- und Ankunftsuniversität lässt sich in Form eines „Abstiegskoeffizienten“ des Typs Rank der Ankunftsuniversität dividiert durch Rank der Ausgangsuniversität abbilden. Je niedriger dieser Koeffizient liegt, desto besser rangiert die Ankunftsuniversität im Vergleich zu der Universität, an der der Abschluss erworben wurde. Ein Koeffizient von 1 signalisiert, dass der betreffende Forscher an einer Universität lehrt, die ebenso hoch eingestuft wird wie die seines Doktorvaters, ein Koeffizient von 3, dass er an einer Universität beschäftigt ist, die nur ein Drittel so hoch rangiert, ein Koeffizient von 0,5 , dass er an einer doppelt so hoch eingestuften Universität arbeitet. Für die Angehörigen der Gruppe A erreicht dieser Koeffizient einen Wert von 10,5, während er für die Ökonomen der Gruppe B alleine nur halb so hoch bei 5,6 liegt.

Offensichtlich sind es also nicht „die Ökonomen“, die sich zugunsten der Todesstrafe aussprechen, während „die Soziologen“ sie ablehnen, sondern ein bestimmter Typus von Ökonomen. 
26. Jahrgang 2015, Heft 1 Sacha Raoult

Tabelle 2: Vergleich zwischen den Rängen der Ausgangsuniversitäten und den Ankunftsuniversitäten der Gruppen A und B.

\begin{tabular}{lcccc} 
& Alle & Gruppe A & $\begin{array}{c}\text { Ökonomen } \\
\text { Gruppe B }\end{array}$ & $\begin{array}{c}\text { übrige Angehörige } \\
\text { Gruppe B }\end{array}$ \\
\hline gesamt & 28 & 10 & 8 & 10 \\
Abschluss in Top 200 & 20 & 8 & 6 & 6 \\
Abschluss in Top 100 & 20 & 8 & 6 & 6 \\
Abschluss in Top 50 & 13 & 4 & 5 & 4 \\
Stelle in Top 200 & 16 & 5 & 5 & 6 \\
Stelle in Top 100 & 10 & 3 & 5 & $0^{*}$ \\
Stelle in Top 50 & 3 & $0^{*}$ & $3^{*}$ & 11.64 \\
Abstiegsindex & 9.50 & 10.42 & 5.67 & \\
\hline
\end{tabular}

${ }^{*} \mathrm{p}<0,05$.

Tatsächlich sind die vorsichtigsten Arbeiten über die Todesstrafe das Werk renommierter Wirtschaftswissenschaftler. John B. Taylor von der Universität Stanford beispielsweise gilt als nobelpreiswürdig; seine Theorien über den Zinssatz werden von zahlreichen Zentralbanken in der ganzen Welt angewandt. Edward Leamer, Erfinder des Sensitivitätstests Extreme Bound Analysis und Professor an der UCLA, ist einer der großen Namen der ökonometrischen Epistemologie. Er war es, der die Forschung wenige Jahre nach dem Erscheinen der ersten Studie Isaac Ehrlichs (1975) vor den Möglichkeiten eines data mining gewarnt hat, wie es die ad hoc-Spezifizierung von Regressionen zum Thema Todesstrafe darstellt, und zwar in einem Aufsatz, der laut Google Scholar 1.800 mal zitiert worden ist und den humorigen Titel „Let's take the Con out of Econometrics" (1982) trägt. Peter Passel war, bevor er sich dem Think-Tank des Milken Institute anschloss, Professor an der Columbia University und Mitglied der Wirtschaftsredaktion der New York Times. Mit anderen Worten, diese drei Ökonomen haben, jeder auf seine Weise, höchste Anerkennung erfahren und an den am höchsten angesehenen Fakultäten der Vereinigten Staaten gelehrt.

Im Gegensatz dazu weist die typische Karrierelinie eines Ökonomen aus der Gruppe A nach unten: Es handelt sich um einen Forscher, dessen letzter Beschäftigungsort im Allgemeinen weitaus weniger Prestige hat als der, wo er studiert hat. Dabei ist der Werdegang dieser Forscher zu Beginn ihrer 
Karriere so unterschiedlich nicht. Der Ausgangspunkt war bei diesen Autoren fast ebenso häufig eine große Universität. Während aber die Mehrzahl der Ökonomen in der Gruppe B, die ihre Doktorarbeit an einer der Top 50Universitäten geschrieben haben, danach an einer Universität des gleichen Rangs unterrichtet haben, wechselte Isaac Ehrlich von Columbia (13) an die University of Buffalo (178), Stephen Layson von der University of Chicago (9) an die University of North Carolina (301-350), Roberto Marchesini von der University of Texas in Austin (27) an die University of Houston (nicht gelistet) und James Yunker von der Northwestern University (22) an die Western Illinois University (nicht gelistet).

Die Resultate hinsichtlich der Todesstrafe werden mit der Ankunftsuniversität korreliert, nicht mit der Ausgangsuniversität. Unser Ergebnis ist aber umso überraschender, als wir selbst innerhalb der Gruppe A die gleiche Aufteilung finden. Die am besten platzierten Ökonomen dieser Gruppe (diejenigen, die an der Emory University reüssiert haben, einer methodistischen Hochschule, die Rang 80 belegt) kommen zwar zu Ergebnissen, die für die Todesstrafe sprechen; diese sind aber weniger extrem. Hier liegt der Grund dafür, dass Schaubild 6 so aufgeräumt wirkt: Die dem Nullwert am nächsten liegenden Resultate entstammen den besten Universitäten, die dem Wert -1 nächsten den am schlechtesten platzierten, während die mittleren Resultate an Universitäten aus dem mittleren Feld produziert werden. Hierher rührt der äußerst starke Koeffizient $\mathrm{R}^{2}$, der darauf hinweist, dass diese Spur trotz der kleinen Stichprobe vielversprechend ist. Wir finden also selbst unter den Ökonomen, die sich für die Todesstrafe aussprechen, eine weitere Unterteilung in diejenigen, die uneingeschränkt dafür sind und diejenigen, die sich ein bisschen nuancierter äußern, wobei diese Unterteilung dem gleichen Kriterium folgt (dem Rang der Universität), was wiederum der Form des Fraktals entspricht, das Andrew Abbott in seiner Geschichte der Sozialwissenschaften (2001) gefunden hat.

Arbeiten, die regelmäßig zugunsten der Todesstrafe argumentieren, zeichnen sich also dadurch aus, dass sie von Akademikern hervorgebracht werden, die den stärksten Abstieg zwischen ihrer Ausgangsuniversität und ihrer letzten Beschäftigung durchlebt haben. Dieses Faktum ist merkwürdig, weil die Professoren und Professorinnen, die sie bei ihren Forschungen betreut haben, ihrerseits an hoch eingestuften Universitäten tätig waren. Ist es also ein Zufall, wenn einige von ihnen ihre Distanz zu solchen Ergebnissen 
wahren? So hat Gary Becker, Nobelpreisträger und bedeutender Professor für Ökonomie in Chicago, als einer der Initiatoren der Bewegung hin zur kriminellen Rationalität und Mentor mehrerer Ökonomen der Gruppe A selbst nie solche Studien verfasst, und er ist weitaus vorsichtiger in seinen Schlussfolgerungen als seine Schüler und Schülerinnen. Während die Angehörigen der Gruppe A betonen, eine abschreckende Wirkung der Todesstrafe sei ,im Wesentlichen“ oder „einstimmig“ von der empirischen Literatur festgestellt worden, schrieb Gary Becker in seinem Blog vor einigen Jahren: „Es unterliegt kaum einem Zweifel, dass diese quantitativen empirischen Arbeiten uneindeutige Ergebnisse liefern" und fügte hinzu: Wenn er persönlich immer vom Abschreckungseffekt der Todesstrafe überzeugt gewesen sei, habe dies auch an philosophischen Erwägungen gelegen, die über das hinausgingen, was in solchen Studien gezeigt werden könne. In einer Konferenz, die kurz vor seinem Tod abgehalten wurde, erklärte Gary Becker erneut, dass „die Resultate dieser Arbeiten [über die Todesstrafe] uneindeutig sind" und dass er es vorzöge, wenn diese Frage die Diskussion seiner Theorien nicht allzu sehr monopolisiere (Becker/Ewald/Harcourt 2013).

Es ist offensichtlich, dass bei der Interpretation unserer Daten Vorsicht angezeigt ist. Zunächst einmal sprechen wir hier von einer kleinen Stichprobe von Forschern (insgesamt 28) in einem kleinen Bereich (65 Publikationen). Die vorläufigen Resultate dieser Studie deuten aber darauf hin, dass es möglich sein müsste, sie für andere stark polarisierte Forschungsbereiche wie die Verbindungen zwischen Gewalt und Feuerwaffen oder die Nützlichkeit versicherungsmathematischer Werkzeuge für die Vorhersage von Rückfallquoten zu reproduzieren (siehe Raoult 2014). Die Studie eröffnet eine ganze Reihe neuer Forschungspisten. So ließe sich die Frage stellen, in welcher Form der Lebensweg der Forscher und ihre Zugehörigkeit zu bestimmten wissenschaftlichen Zirkeln die Ergebnisse beeinflusst, die sie erhalten. Es ließe sich fragen, ob bestimmte Resultate nur durch Arbeiten minderer Qualität erzielt werden können, die - sofern es gelingt, sie in einer angesehenen Zeitschrift unterzubringen - einer Überprüfung durch Berufungskommissionen großer Universitäten nicht standhalten würden, was wiederum daran liegen könnte, dass eine solche Forschung seit der vernichtenden Kritik an den Arbeiten Isaac Ehrlichs in den 1970er Jahren (z. B. 1975, 1977) in den Ruf geraten ist, nicht seriös zu sein. Es ließe sich dann untersuchen, in welchem Maße es Trends bei der Rekrutierung seitens der amerikanischen Uni- 
Empirische Sozialforschung und ihre Macher

26. Jahrgang 2015, Heft 1

versitäten gibt oder auch eine Korrelation zwischen bestimmten ökonometrischen Resultaten und bestimmten politischen Haltungen. Es gibt kurz gesagt Diskussionsbedarf über die Wechselwirkungen zwischen den Markern akademischen „Prestiges“ - das bereits mehrfach in bemerkenswerter Weise theoretisiert worden ist (z.B. Bourdieu 1984; Latour/Woolgar 2006) - und den empirischen Ergebnissen über einen bestimmten Gegenstand. Eine andere Richtung bestünde darin, eine Verbindung zu ziehen zwischen den jeweiligen Resultaten und der Diversität der akademischen Kulturen oder den Unterschieden zwischen den Disziplinen.

Sicher ist, dass sich die allgemeinen Tendenzen, die wir ans Licht gebracht haben, in dem Maße verstärken, in dem wir den Wissenschaftlern die Kontrolle über ihre eigenen Resultate überlassen, das heißt wenn sich das Interesse eher auf die Schlussfolgerungen denn auf die Rohdaten richtet. Die Effektstärken aus Arbeiten über die Todesstrafe korrelieren im Allgemeinen mit den Effektstärken aus den vorherigen Arbeiten des gleichen Forschers und mit dem Rang der Ankunftsuniversität, wobei sich diese Korrelation für die korrigierten Effektstärken sowie noch weiter für einzelne Arbeiten mit Kontrollvariablen verstärkt.

\section{Schlussfolgerung}

Was machen wir mit diesem Ergebnis? Die erste Aufgabe besteht darin, zu klären, über welche Werkzeuge die wissenschaftliche Welt verfügt, um zu verhindern, dass „Autorenbias“ das Untersuchungsfeld verzerren. Hier gibt es zunächst die Ebene der Zeitschriften, die aber nicht in der Lage ist, das hier angesprochene Problem zu lösen. Selbst wenn die Autoren der am stärksten zugunsten der Todesstrafe sprechenden Resultate an weniger angesehenen Fakultäten lehren, bedeutet dies nicht, dass ihre Arbeiten in der grauen Literatur veröffentlicht würden. Wir haben es mit der American Economic Review, dem Journal of Criminal Justice oder der American Law and Economic Review zu tun. Allerdings trifft es zu, dass die fehlende öffentliche Zugänglichkeit von Datenbasen eine nachträgliche Kritik ohne Zuarbeit seitens der Autoren erschwert, was angesichts stark mathematisierter und undurchsichtiger Arbeiten einigermaßen problematisch ist.

Was Metaanalysen angeht, so gibt es seit mehr als einem Jahrzehnt mehrere Initiativen, um zu vermeiden, dass diese systematischen Analysen eines 
Feldes nicht von Publikationstrends verzerrt werden. Ein Autor hat eine Reihe von Tests vorgeschlagen, mit denen sich statistisch anormale Resultate seit dem Ende der 1990er Jahre auffinden lassen, um Betrug oder das Unterdrücken von Daten in Forschungen aufzudecken, die von der pharmazeutischen Industrie finanziert wurden (Egger et al. 1997). Es ist bedauerlich, dass dieser Test nicht in hinreichendem Umfang angewendet wird, denn er ist in jedem Bereich nützlich. Die Campbell Corporation schlägt eine äußerst wirksame Methode zum Ausschluss unsicherer oder verdächtiger Studien vor. Jean-Claude Combessie zeigt am Beispiel der Ungleichheit von Lebensschicksalen, dass man wenigstens jedes neue Resultat unter Anwendung aller verfügbaren Analysemethoden präsentieren könnte, um so den Leser und die Leserin nicht über die Vielfalt der methodologischen Wahlmöglichkeiten im Unklaren zu lassen, zumal diese zu unterschiedlichen Resultaten führen (Combessie 2001). Im Anschluss an unsere Arbeit ließe sich jeder Metaanalyse ein Test „Resultat je Autorengruppe“ hinzufügen, um Forschungsgruppen erkennbar zu machen, die immer wieder zu den gleichen Resultaten kommen, sei es dass dieser Trend mit Finanzierungen, einer Methodologie, einer Ideologie verbunden ist oder sich auf die Frage nach dem konkreten Forschungsbereich reduziert. Auf diese Weise könnte dem Umstand Genüge getan werden, dass die „wissenschaftliche Wahrheit“ nicht mehr ist als ein „Mittelwert“ der Resultate, die von den verschiedenen Forschergemeinschaften erbracht werden.

Ein letzter Fragenkomplex bleibt zu klären; er betont die Bedeutung einer gründlichen Kenntnis der einschlägigen Debatten und der Geschichte des Feldes. Von Thorsten Sellin (1959) bis hin zum Vergleich zwischen Hong Kong und Singapur (Zimring/Fagan/Johnson 2010) hat die Entwicklung der Tötungsraten in demographisch ähnlichen Jurisdiktionen mit stark unterschiedlichen Politiken hinsichtlich der Todesstrafe immer den Eindruck erweckt, dass es einen Nulleffekt der Hinrichtungen auf die Tötungsdelikte gebe. Mit anderen Worten: Wenn zwei geographische Zonen Raten von Tötungsdelikten haben, die sich parallel entwickeln, und eine der beiden Zonen ändert ihre Politik hinsichtlich der Todesstrafe drastisch, verhindert dies nicht, dass die Tötungsraten sich weiterhin parallel entwickeln. Man könnte nun untersuchen, ob es Zufall ist, dass dieses Bruttoresultat, für das man keiner höheren Mathematik bedarf, ebenfalls einer heterogenen Mehrheitsforschung sowie der an den angesehensten Universitäten angestrengten For- 
schung entspricht, während die quantitative Forschung, der es gelingt, diese Bruttodaten umzudrehen, minoritär ist und von einer kleinen, sehr homogenen und randständigen Gruppe innerhalb der Universität produziert wird. Meine derzeitige Hypothese lautet, dass das, was man gemeinhin mit akademischem Prestige verknüpft, oft einhergeht mit einem transdisziplinären und internationalen wissenschaftlichen Dialog, der zu einer sorgfältigeren Aufarbeitung von Meinungen zwingt sowie dazu, immer wieder auf grundlegendes Wissen zurückzugreifen (in diesem Fall auf die Sellin-Kurven).

\section{Anmerkungen}

1 Maître de conférences an der Universität Aix-Marseille (AMU, LDPSC EA 4690), Andrew W. Mellon-Fellow an der Universität von Chicago. Danksagungen: Der Autor möchte sich für Ratschläge und Anmerkungen bedanken bei den anonymen Lektoren von Déviance \& Société, da diese stark dazu beigetragen haben, die Klarheit der folgenden Ausführungen zu steigern, sowie bei Philippe Combessie, Bernard Harcourt, Laurent Muchielli, Andrew Abbott und den Mitgliedern der Groupe Européen de Recherches sur les Normalités (GERN), die bei der ursprünglichen Vorstellung meiner Ergebnisse auf dem Kolloquium des GERN am 21. Juni 2013 anwesend waren, namentlich Axel Grönemeyer und Laurent Mucchielli.

2 Alle Zitate im Text wurden vom Autor übersetzt. Der an einem tieferen Einblick in die Ergebnisse dieser Arbeit interessierte Leser wird eine Liste der hier analysierten Studien in den Kategorien I, II und III bei Yang und Lester (2008) finden, wobei die Untergruppe der hinsichtlich von Autorenbias untersuchten Studien jenen 64 Arbeiten entspricht, die von Forschern und Forscherinnen stammen, die mindestens zwei Veröffentlichungen aus dieser Liste hervorgebracht haben, wodurch ihre Arbeiten miteinander verglichen werden können. Die genauen Literaturangaben der in dieser Untersuchung verwendeten Studien finden sich in Yang/Lester (2008); eine Bibliographie dieser Arbeiten kann auch direkt beim Autor angefragt werden: [sraoult @uchicago.edu].

3 Laut Yang und Lester (2008) machten 2008 solche Arbeiten 85 Prozent der quantitativen Studien über den abschreckenden Effekt der Todesstrafe aus; sie stützten sich auf Zeitreihen- oder geographische Daten sowie Paneldaten. 5 Prozent der Arbeiten betrachteten die Entwicklung der Raten von Tötungsdelikten in den Tagen nach einer Hinrichtung und 10 Prozent stellen eine Verbindung zwischen der medialen Verarbeitung einer Exekution und der Zahl der Morde her.

4 Es handelt sich hier um Donohue und Wolfer (2006), eine Sensibilitätsanalyse, in der mehrere Arbeiten einer kritischen Überprüfung unterzogen werden, die sich für die Todesstrafe aussprechen; sie wird im Gutachten von Jeffrey Fagan zitiert.

5 Zur Klassierung einer Person haben wir das Ranking der Universität der letzten Beschäftigung in der Times Higher Education von 2013-2014 verwendet, für die Klassierung einer Forschergruppe haben wir die bestklassierte Universität unter den letzten Beschäftigungen der Gruppenmitglieder eingesetzt. Es handelt sich dabei um ein Ranking der obersten 400. Nicht aufgeführte Universitäten werden als 401. Rang gewertet. Für die Universitäten der 
Ränge 200 bis 400 gruppiert das Ranking der Times Higher Education in Gruppen von 50 (beispielsweise 301-350); wir haben also einen Mittelwert der Klassierung (also in diesem Falle 325,5) verwendet.

6 Wenn man die aktuell bekleideten Stellen anstelle der letzten Beschäftigung berücksichtigt und auf diese Weise diejenigen nicht zählt, die die akademische Welt verlassen haben, verlieren die Ökonomen der Gruppe B eine Position in den Top 50, und das Resultat verliert seine Quantifizierbarkeit.

\section{Literatur}

Abbott, Andrew, 2001: Chaos of disciplines. Chicago: University of Chicago Press.

Barnes, Barry/Bloor, David/Henry, John (eds.), 1996: Scientific knowledge. A sociological analysis. Chicago: University of Chicago Press.

Beccaria, Cesare, 1998: Über Verbrechen und Strafen (it. org. 1851). Frankfurt/M.: Insel.

Becker, Gary S., 1968: Crime and Punishment: An Economic Approach. Journal of Political Economy 76/2: 169-217.

Becker, Gary S./Ewald, François/Harcourt, Bernard E., 2013: Becker and Foucault on Crime and Punishment. University of Chicago Coase-Sandor Institute for Law \& Economics Research Paper 654). Internetquelle: [http://chicagounbound.uchicago.edu/law_and_economics/ 635/].

Bekelman, Justin E./Li, Yan/Gross, Cary P., 2003: Scope and impact of financial conflicts of interest in biomedical research: A systematic review. Journal of the American Medical Association (Jama) 289/4: 454-465.

Bourdieu, Pierre, 1984: Homo academicus. Paris: Édition de Minuit [deutsche Ausgabe 1988: Homo academicus. Frankfurt/M.: Suhrkamp].

Bresnick, Stephen D., 2008: Does extensive financial support create author bias? The Juvéderm Injectable Gel Study. Aesthetic Surgery Journal 28/5: 596-597.

Combessie, Jean-Claude, 2011: Analyse critique d'une histoire des traitements statistiques des inégalités de destin. Actes de la recherche en sciences sociales 3: 4-31.

Connecticut Judiciary Committee, 2011: An act repealing the death penalty. Joint Favorable Report, Bill No.: SB-1035, Vote Date: 4/12/2011. Internetquelle: [https://www.cga.ct.gov/ 2011/JFR/S/2011SB-01035-R00JUD-JFR.htm].

Dölling, Dieter/Entorf, Horst/Hermann, Dieter/Rupp, Thomas, 2009: Is deterrence effective? Results of a meta-analysis of punishment. European Journal on Criminal Policy and Research 15/1-2: 201-224.

Donohue, John J./Wolfers, Justin J., 2006: Uses and abuses of empirical evidence in the death penalty debate. Stanford Law Review 58/1: 791-846.

Donohue, John J./Ayres, Ian, 2009: More guns, less crime fails again: The latest evidence from 1977-2006. Econ Journal Watch 6/2: 218-238.

Durlauf, Steven N./Fu, Chao/Navarro, Salvador, 2013: Capital punishment and deterrence: Understanding disparate results. Journal of Quantitative Criminology 29/1: 103-121.

Egger, Matthias/Smith, George Davey/Schneider, Martin/Minder, Christoph, 1997: Bias in metaanalysis detected by a simple, graphical test. Biomedical Journal 315/7109: 629-634. 
Ehrlich, Isaac, 1975: The deterrent effect of capital punishment: A question of life and death. American Economic Review 65: 397-417.

Ehrlich, Isaac, 1977: Capital punishment and deterrence: Some further thoughts and additional evidence. Journal of Political Economy 85: 741-788.

Foucault, Michel, 2014: Les mots et les choses. Une archéologie des sciences humaines [org. 1966]. Paris: Gallimard [deutsch 1974: Die Ordnung der Dinge. Eine Archäologie der Humanwissenschaften. Frankfurt/M.: Suhrkamp].

Gerritzen, Berit/Kirchgässner, Gebhard, 2013: Facts or Ideology: What determines the results of econometric estimates of the deterrence effect of death penalty? A Meta-Analysis. CESifo Working Paper No. 4159. Internetquelle : [http://papers.ssrn.com/sol3/papers.cfm?abstract $\mathrm{id}=2243448$.

Gide, André, 2012: Les nourritures terrestres/Les nouvelles nourritures. Paris: Gallimard.

Goertzel, Ted/Goertzel, Benjamin, 2008: Capital punishment and homicide rates: Sociological realities and econometric distortions. Critical Sociology 34/2: 239-254.

Helmus, Leslie/Hanson, R. Karl/Thornton, David/Barchishin, Kelly M./Harris, Andrew Jr., 2012: Absolute recidivism rates predicted by Static-99R and Static-2002R Sex Offender Risk Assessment Tools vary across samples. A meta-analysis. Criminal Justice and Behavior 39/9: 1148-1171.

Jonson, Cheryl Lero, 2010: The impact of imprisonment on reoffending: A meta-analysis. Diss. University of Cincinnati.

Kirchgässner, Gebhard, 2011: Econometric estimates of deterrence of the death penalty: Facts or ideology?" Kyklos 64.3: 448-478.

Kuhn, Thomas S., 1967: Die Struktur wissenschaftlicher Revolutionen. Frankfurt/M.: Suhrkamp [am. org. 1962: The Structure of Scientific Revolutions. Chicago: University of Chicago Press].

Latour, Bruno/Woolgar, Steve, 2006 : La vie de laboratoire. La production des faits scientfiques. Paris: La découverte.

Leamer, Edward E., 1983: Let's take the con out of econometrics. The American Economic Review 73/1: 31-43.

Lipsey, Mark W./Cullen, Francis T., 2007: The effectiveness of correctional rehabilitation: A review of systematic reviews. Annual Review of Law \& Social Science 3: 297-320.

Liu, Zhiqiang, 2004: Capital punishment and the deterrence hypothesis: Some new insights and empirical evidence. Eastern Economic Journal 30/2: 237-258.

McManus, Walter S., 1985: Estimates of the deterrent effect of capital punishment: The importance of the researcher's prior beliefs. The Journal of Political Economy 93/2: 417-425.

Paternoster, Raymond, 2010: How much do we really know about criminal deterrence. Journal of Criminal Law \& Criminology 100: 765.

Pratt, Travis C./Cullen, Francis T., 2005: Assessing macro-level predictors and theories of crime: A meta-analysis. Crime and Justice 32: 373-450.

Raoult, Sacha, 2014: Picking on Piketty. An Analysis of the Reception of Thomas Piketty's Capital in the Twenty First Century: On Social Status and Academic Standing“. Columbia Center for Contemporary Critical Thought, 1 Dec. 2014. Internetquelle: [http://web.law.co lumbia.edu/contemporary-critical-thought/events/past-events].

Rubin, Paul, 2006: Statistical evidence on capital punishment and the deterrence of homicide. Written testimony for the Senate Judiciary Committee on the Constitution, Civil Rights, and Property Rights, February 1, 2006. Washington [Internetquelle: http://www.judiciary. senate.gov/download/2006/02/01/paul-rubin-testimony-020106]. 
Shepherd, Joanna M., 2005a: Deterrence versus brutalization: Capital punishment's differing impacts among states. Michigan Law Review 104: 203-255.

Shepherd, Joanna M., 2005b: Why not all executions deter murder. Christian Science Monitor, December 14. Internetquelle: [http://www.csmonitor.com/2005/1214/p09s01-coop.html].

Sellin, Thorsten, 1959: The death penalty. Philadelphia: American Law Institute.

Thompson, Dennis F., 1993: Understanding financial conflicts of interest. New England Journal of Medicine 329: 573-573.

Vaccaro, Alexander R./Patel, Alpesh A./Fisher, Charles, 2011: Author conflict and bias in research: Quantifying the downgrade in methodology. Spine 36/14: E895-E896.

Yang, Bijou, 1998: Economic analyses of the deterrent effect of the death penalty. Pp. 83-105 in: Lester, D. (ed.), The death penalty ( $2^{\text {nd }}$ edition). Springfield, IL: Charles C. Thomas.

Yang, Bijou/Lester, David, 2008: The deterrent effect of executions: A meta-analysis thirty years after Ehrlich. Journal of Criminal Justice 36/5: 453-460.

Zimring, Franklin E./Fagan, Jeffrey/Johnson, David T., 2010: Executions, deterrence, and homicide: A tale of two cities. Journal of Empirical Legal Studies 7/1: 1-29. 


\title{
Empirical Social Research and its Producers \\ The Social Production of Knowledge about the Effects of Capital Punishment
}

\begin{abstract}
Rational choice theory has given birth to a massive quantitative production of empirical knowledge on the deterrent effect of punishment, a field in which results are often confusing and discordant. This paper explores the research communities working on the correlation between executions and homicides, and identifies a "vocal minority" of academics that are very favorable to the death penalty. Knowing the name of the author of an article is a better predictor of the correlations found by the article that the type of data used. This study also shows a profile of the author who has a large amount of pro-death penalty result: it is an American economist, who does not present himself as a specialist in crime and punishment, and who teaches in a faculty ranked lower than the one where he obtained his PhD. On the other hand, sociologists and economists of high-rank universities tend to find results closer to a "zero" effect of the death penalty on homicide.
\end{abstract}

Sacha Raoult

Universität Aix-Marseille Laboratoire de droit privé et de sciences criminelles 3 avenue Robert-Schuman 13628 Aix-en-Provence Cedex 1 sacha.raoult@univ-amu.fr 